\title{
Liver enzyme inflames fat
}

Obesity-induced activation of adipose tissue inflammation is closely correlated with insulin resistance, but the mechanisms mediating such pathological inflammation are poorly understood. Writing in Nature, Tabas, Ozcan and colleagues report that in mice, obesity stimulates hepatocytes to secrete the enzyme dipeptidyl peptidase 4 (DPP4), which promotes adipose tissue inflammation and insulin resistance, highlighting liver DPP4 as an attractive antidiabetic target. Although oral DPP4 inhibitors are currently used to treat type 2 diabetes, they target gut endothelial DPP4 to lower blood sugar and do not affect insulin resistance.

In obesity, a pathway in hepatocytes involving calcium/ calmodulin-dependent kinase type II (CaMKII) is involved in increased hepatic glucose production and impaired hepatic insulin signalling. Previous studies have reported that hepatocyte-specific deletion of CaMKII in diet-induced obese (DIO) mice (CaMKII(KO) mice) improves glucose and insulin tolerance.

Tabas, Ozcan and colleagues first noted that these CaMKII(KO) mice also exhibited reduced inflammatory adipose tissue macrophages (ATMs) and levels of inflammatory cytokines in visceral adipose tissue (VAT) compared with wild-type DIO mice. They therefore hypothesized that in obesity,

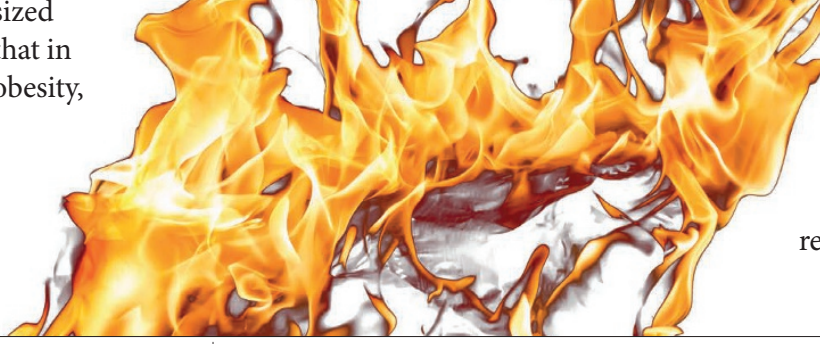

activation of liver CaMKII signalling results in the secretion of a circulatory factor that promotes inflammation in VAT.

To test their hypothesis, the authors first carried out ex vivo studies in which cells from the stromal vascular fraction (SVF) of VAT were incubated with mouse plasma. Plasma from DIO mice induced higher expression of the inflammatory markers MCP1 and interleukin (IL)-6 than plasma from lean mice in SVF cells from DIO mice, but did not have this effect on SVF cells from lean mice. In contrast, plasma from CaMKII(KO) mice had little effect on MCP1 or IL-6 expression. Similar results were observed using adipose tissue macrophages from obese mice.

Liquid chromatography and mass spectrometry studies of DIO mouse plasma identified DPP4 as the factor likely responsible for the induction of MCP1. Indeed, the DPP4 inhibitor KR62436 suppressed induction of inflammatory markers in SVF cells by DIO mouse plasma. In addition, DPP4 activity in the plasma and DPP4 mRNA expression in the liver of mice correlated precisely with VAT inflammation.

Furthermore, infection of DIO mice with an adeno-associated virus encoding a DPP4-targeted short hairpin RNA significantly decreased plasma DPP4 and reduced levels of inflammatory cytokine expression and ATMs in VAT. The infected mice also exhibited improved glucose homeostasis and insulin signalling in VAT and the liver. Similar beneficial effects of such DPP4 reduction were observed in $o b / o b$ mice.

Next, the authors compared the effects of an oral DPP4 inhibitor sitagliptin with silencing of hepatocyte DPP4 in DIO mice. While both treatments lowered blood glucose and improved oral glucose tolerance, only reduction of hepatocyte DPP4 reduced VAT inflammation and decreased insulin resistance.

Finally, the authors investigated the mechanism of DPP4-induced VAT macrophage inflammation. Further analysis of DIO mouse plasma and studies in DIO mice revealed that an additional plasma factor - factor Xa (FXa) - was needed for DPP4 to promote SVF inflammation. DPP4 and FXa synergistically activated inflammatory signalling in mouse and human macrophages, mediating their effects through caveolin 1 (CAV1) and proteinase-activated receptor 2 (PAR2), respectively. In $o b / o b$ mice, small interfering RNA-mediated silencing of CAV1 or PAR2 in ATMs suppressed VAT inflammation and reduced insulin resistance.

These findings emphasize the important role of crosstalk between hepatocytes and adipose tissue in metabolic disease and suggest that silencing of hepatocyte DPP4 or inhibiting the action of DPP4 or FXa on macrophages may represent new approaches to reducing insulin resistance in type 2 diabetes. The laboratory is currently investigating why inhibiting DPP4 via oral inhibitors does not block fat inflammation and improve metabolism despite inhibiting plasma DPP4.

Sarah Crunkhorn

ORIGINAL ARTICLE Ghorpade, D. S. et al. Hepatocyte-secreted DPP4 in obesity promotes adipose inflammation and insulin resistance. Nature 555, 673-677 (2018) 